\title{
Focusing and Polarization in Intuitionistic Logic
}

\author{
Chuck Liang ${ }^{1}$ and Dale Miller ${ }^{2}$ \\ 1 Department of Computer Science, Hofstra University, Hempstead, NY 11550 \\ chuck.liang at hofstra.edu \\ 2 INRIA \& LIX/Ecole Polytechnique, Palaiseau, France \\ dale.miller at inria.fr
}

\begin{abstract}
A focused proof system provides a normal form to cut-free proofs that structures the application of invertible and non-invertible inference rules. The focused proof system of Andreoli for linear logic has been applied to both the proof search and the proof normalization approaches to computation. Various proof systems in literature exhibit characteristics of focusing to one degree or another. We present a new, focused proof system for intuitionistic logic, called $L J F$, and show how other proof systems can be mapped into the new system by inserting logical connectives that prematurely stop focusing. We also use $L J F$ to design a focused proof system for classical logic. Our approach to the design and analysis of these systems is based on the completeness of focusing in linear logic and on the notion of polarity that appears in Girard's LC and LU proof systems.
\end{abstract}

\section{Introduction}

Cut-elimination provides an important normal form for sequent calculus proofs. But what normal forms can we uncover about the structure of cut-free proofs? Since cut-free proofs play important roles in the foundations of computation, such normal forms might find a range of applications in the proof normalization foundations for functional programming or in the proof search foundations of logic programming.

\subsection{About focusing}

Andreoli's focusing proof system for linear logic (the triadic proof system of [1]) provides a normal form for cut-free proofs in linear logic. Although we describe this system, here called $L L F$, in more detail in Section 2, we highlight two aspect of focusing proofs here. First, linear logic connectives can be divided into the asynchronous connectives, whose right-introduction rules are invertible, and the synchronous connectives, whose right introduction rules are not (generally) invertible. The search for a focused proof can capitalize on this classification by applying (reading inference rules from conclusion to premise) all invertible rules in any order (without the need for backtracking) and by applying a chain of non-invertible rules that focus on a given formula and its positive subformulas. 
Such a chain of applications, usually called a focus, terminates when it reaches an asynchronous formula. Proof search can then alternate between applications of asynchronous introduction rules and chains of synchronous introduction rules.

A second aspect of focusing proofs is that the synchronous/asynchronous classification of non-atomic formulas must be extended to atomic formulas. The arbitrary assignment of positive (synchronous) and negative (asynchronous) bias to atomic formulas can have a major impact on, not the existence of focused proofs, but the shape of focused proofs. For example, consider the Horn clause specification of the Fibonacci series:

$$
f i b(0,0) \wedge f i b(1,1) \wedge \forall n \forall f \forall f^{\prime}\left[f i b(n, f) \wedge f i b\left(n+1, f^{\prime}\right) \supset f i b\left(n+2, f+f^{\prime}\right)\right] .
$$

If all atomic formulas are given negative bias, then the only focused proofs of fib $\left(n, f_{n}\right)$ are those that can be classified as "backward chaining" (the size of the smallest one being exponential in $n$ ). On the other hand, if all atomic formulas are given positive bias, then the only focused proofs are those that can be classified as "forward chaining" (the size of the smallest one being linear in $n$ ).

\subsection{Results}

The contributions of this paper are the following. First, we introduce in Section 5 a new focusing proof system $L J F$ and show that it is sound and complete for intuitionistic logic. Notable features of LJF are that it allows for atoms of different bias and it contains two versions of conjunction: while these conjunctions are logically equivalent, they are affected by focusing differently. Second, in Section 6, we show how several other focusing proof systems can be captured in $L J F$, in the sense of full completeness (one-to-one correspondence between proofs in different systems). One should note that while there are many focusing proof systems for intuitionistic logic in the literature, we appear to be the first to provide a single (intuitionistic) framework for capturing many of them. Third, in Section 7, we use $L J F$ to derive $L K F$, a focusing system for classical logic.

\subsection{Methodology and Related work}

There are a number of sequent calculus proof systems known to be complete for intuitionistic logic that exhibit characteristics of focusing. Some of these proof systems are based on fixing globally on either forward chaining or backward chaining. The early work on uniform proofs [17] and the LJT proof system [11] are both backward chaining calculi (all atoms have negative bias). The $L J Q$ calculus $[11,7]$ similarly selects the global preference to be forward chaining (all atoms have positive bias). Less has been published about systems that allow for mixing bias on atoms. The $\lambda$ RCC proof system of Jagadeesan, Nadathur, and Saraswat [13] allows for both forward chaining and backward chaining in a superset of the hereditary Harrop fragment of intuitionistic logic. Chaudhuri, Pfenning, and Price in [3] observed that focusing proofs with mixed biases on atoms can form a declarative basis for mixing forward and backward chaining 
within Horn clauses. The $\mathrm{PhD}$ theses of Howe [12] and Chaudhuri [2] also explored various focusing proof systems for linear and intuitionistic logic.

We are interested in providing a flexible and unifying framework that can collect together important aspects of many of these proof systems. There are several ways to motivate and validate the design of such a system. One approach stays entirely within intuitionistic logic and works directly with invertibility and permutability of inference rules. Such an approach has been taken in many papers, such as $[17,18,7]$. Our approach uses linear logic, with its exponential operators ! and ?, as a unifying framework for looking at intuitionistic (and classical) logic. The fact that Andreoli's focused system was defined for full linear logic provides us with a convenient platform for exploring the issues around focusing and polarity. We translate intuitionistic logic into linear logic, then show that proof systems for intuitionistic logic match focused proofs of the translated image (Section 3). A crucial aspect of understanding focusing in intuitionistic logic is provided by identifying the precise relationship between Andreoli's notion of polarity with Girard's notion of polarity found in the LC [9] and LU [10] systems (Section 4).

Another system concerning polarity and focusing is found in the work of Danos, Joinet and Schellinx [5,6]. Many techniques that they developed, such as inductive decorations, are used throughout our analysis. Our work diverges from theirs in the adaptation of Andreoli's system (LLF) as our main instrument of construction. The $L K_{p}^{\eta}$ system of [6] describes focused proofs for classical logic. Its connections to polarization and focusing were further explored and extended by Laurent, Quatrini and de Falco [14] using polarized proof nets. It may be tempting to speculate that the best way to arrive at a notion of intuitionistic focusing is by simple modifications to these systems, such as restricting them to single-conclusion sequents. Closer examination however, reveal intricate issues concerning this approach. For example, the notion of classical polarity appears to be distinct from and contrary to intuitionistic polarity, especially at the level of atoms (see Sections 4 and 7). Resolving this issue would be central to finding systems that support combined forward and backward chaining. Although the relationship between $L K_{p}^{\eta}$ and our systems is interesting, we chose for this work to derive intuitionistic focusing from focusing in linear logic as opposed to classical logic.

Much of the research into focusing systems has been motivated by their application. For example, the papers $[13,17,12,2]$ are motivated by foundational issues in logic programming and automated deduction. The papers $[11,5,6,14]$ are motivated by foundational issues in functional programming and the $\lambda$-calculus. Also, Levy [15] presents focus-style proof systems for typing in the $\lambda$-calculus and Curien and Herbelin [4] (among others) have noted the relationship between forward chaining and call-by-value evaluation and between backward chaining and call-by-name evaluation.

Our work can be extended to second order logic, although this paper is concerned mainly with first-order quantification.

Many details missing from this paper can be found in the report [16]. 


\section{$2 \quad$ Focusing in Linear Logic}

We summarize the key results from [1] on focusing proofs for linear logic.

A literal is either an atomic formula or the negation of an atomic formula. A linear logic formula is in negation normal form if it does not contain occurrences of $\multimap$ and if all negations have atomic scope. If $K$ is literal, then $K^{\perp}$ denotes its complement: in particular, if $K$ is $A^{\perp}$ then $K^{\perp}$ is $A$.

Connectives in linear logic are either asynchronous or synchronous. The asynchronous connectives are $\perp, \not{8}, ?, \top, \&$, and $\forall$ while the synchronous connectives are their de Morgan dual, namely, 1, $\otimes$, !, 0, $\oplus$, and $\exists$. Asynchronous connectives are those where the right-introduction rule is always invertible. Formally, a formula in negation normal form is of three kinds: literal, asynchronous (i.e., its top-level connective is asynchronous), and synchronous (i.e., its top-level connective is synchronous).

$$
\begin{aligned}
& \frac{\Psi: \Delta \Uparrow L}{\Psi: \Delta \Uparrow \perp, L}[\perp] \quad \frac{\Psi: \Delta \Uparrow F, G, L}{\Psi: \Delta \Uparrow F \not \& G, L}[28] \quad \frac{\Psi, F: \Delta \Uparrow L}{\Psi: \Delta \Uparrow ? F, L}[?]
\end{aligned}
$$

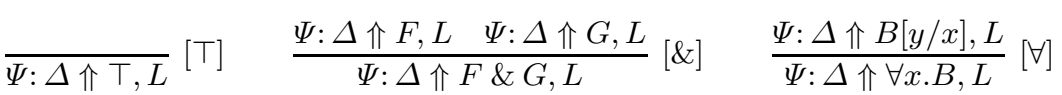

$$
\begin{aligned}
& \frac{\Psi: \Delta, F \Uparrow L}{\Psi: \Delta \Uparrow F, L}[R \Uparrow] \text { provided that } \mathrm{F} \text { is not asynchronous } \\
& \overline{\Psi: \cdot \Downarrow \mathbf{1}}[\mathbf{1}] \quad \frac{\Psi: \Delta_{1} \Downarrow F \quad \Psi: \Delta_{2} \Downarrow G}{\Psi: \Delta_{1}, \Delta_{2} \Downarrow F \otimes G}[\otimes] \quad \frac{\Psi: \Uparrow \uparrow}{\Psi: \cdot \Downarrow ! F}[!] \\
& \frac{\Psi: \Delta \Downarrow F_{1}}{\Psi: \Delta \Downarrow F_{1} \oplus F_{2}}\left[\oplus_{l}\right] \quad \frac{\Psi: \Delta \Downarrow F_{2}}{\Psi: \Delta \Downarrow F_{1} \oplus F_{2}}\left[\oplus_{r}\right] \quad \frac{\Psi: \Delta \Downarrow B[t / x]}{\Psi: \Delta \Downarrow \exists x . B}[\exists] \\
& \frac{\Psi: \Delta \Uparrow F}{\Psi: \Delta \Downarrow F}[R \Downarrow] \quad \text { provided that } F \text { is either asynchronous or a negative literal } \\
& \text { If } K \text { a positive literal: } \overline{\Psi: K^{\perp} \Downarrow K}\left[I_{1}\right] \quad \overline{\Psi, K^{\perp}: \cdot \Downarrow K}\left[I_{2}\right]
\end{aligned}
$$

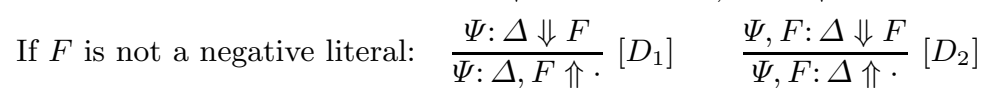

Fig. 1. The focused proof system LLF for linear logic

As mentioned in Section 1.1, the classification of non-atomic formulas as asynchronous or synchronous is pushed to literals by assigning a fixed but arbitrary bias to atoms: an atom given a negative bias is linked to asynchronous behavior while an atom given positive bias is linked to synchronous behavior. In Andreoli's original presentation of LLF [1] all atoms were classified as "positive" and their negations "negative." Girard made a similar assignment for LC [9]. In a classical setting, such a choice works fine since classical negation simply flips bias. In intuitionistic systems, however, a more natural treatment is to assign an arbitrary bias directly to atoms. This bias of atoms is extended to literals: 


\begin{tabular}{|c||c|c|c|}
\hline$B$ & $B^{1}$ & $B^{0}$ & $\left(B^{0}\right)^{\perp}$ \\
\hline \hline atom $Q$ & $Q$ & $Q$ & $Q^{\perp}$ \\
\hline true & 1 & $\top$ & 0 \\
\hline false & 0 & 0 & $\top$ \\
\hline$P \wedge Q$ & $!\left(P^{1} \& Q^{1}\right)$ & $! P^{0} \& ! Q^{0}$ & $?\left(P^{0}\right)^{\perp} \oplus ?\left(Q^{0}\right)^{\perp}$ \\
\hline$P \vee Q$ & $! P^{1} \oplus ! Q^{1}$ & $! P^{0} \oplus ! Q^{0}$ & $?\left(P^{0}\right)^{\perp} \& ?\left(Q^{0}\right)^{\perp}$ \\
\hline$P \supset Q$ & $!\left(?\left(P^{0}\right)^{\perp} \not 8 Q^{1}\right)$ & $! P^{1} \multimap ! Q^{0}$ & $! P^{1} \otimes ?\left(Q^{0}\right)^{\perp}$ \\
\hline$\neg P$ & $!\left(0>? ?\left(P^{0}\right)^{\perp}\right)$ & $! P^{1} \multimap 0$ & $! P^{1} \otimes \top$ \\
\hline$\exists x P$ & $\exists x ! P^{1}$ & $\exists x ! P^{0}$ & $\forall x ?\left(P^{0}\right)^{\perp}$ \\
\hline$\forall x P$ & $! \forall x P^{1}$ & $\forall x ! P^{0}$ & $\exists x ?\left(P^{0}\right)^{\perp}$ \\
\hline
\end{tabular}

Table 1. The $0 / 1$ translation used to encode LJ proofs into linear logic.

negating a negative atom yields a positive literal and negating a positive atom yields a negative literal.

The focusing proof system LLF for linear logic, presented in Figure 1, contains two kinds of sequents. In the sequent $\Psi: \Delta \Uparrow L$, the "zones" $\Psi$ and $\Delta$ are multisets and $L$ is a list. This sequent encodes the usual one-sided sequent $\vdash$ ? $\Psi, \Delta, L$ (here, we assume the natural coercion of lists into multisets). This sequent will also satisfy the invariant that requires $\Delta$ to contain only literals and synchronous formulas. In the sequent $\Psi: \Delta \Downarrow F$, the zone $\Psi$ is a multiset of formulas and $\Delta$ is a multiset of literals and synchronous formulas, and $F$ is a single formula. Notice that the bias of literals is explicitly referred to in the $[R \Uparrow]$ and initial rules: in particular, in the initial rules, the literal on the right of the $\Downarrow$ must be positive.

Changes to the bias assigned to atoms does not affect provability of a linear logic formula: instead it affects the structure of focused proofs.

\section{Translating Intuitionistic Logic}

Table 1 contains a translation of intuitionistic logic into linear logic. This translation induces a bijection between arbitrary LJ proofs and LLF proofs of the translated image in the following sense. First notice that this translation is asymmetric: the intuitionistic formula $A$ is translated using $A^{1}$ if it occurs on the right-side of an LJ sequent and as $A^{0}$ if it occurs on the left-side. Since this translation is used to capture cut-free proofs, such distinctions are not problematic. Since the left-hand side of a sequent in LJ will be negated when translated to a one-sided linear logic sequent, $\left(B^{0}\right)^{\perp}$ is also shown. The following Proposition essentially says that, via this translation, linear logic focusing can capture arbitrary proofs in LJ. Here, $\vdash_{I}$ denotes an intuitionistic logic sequent.

Proposition 1. Let $\left(\Gamma^{0}\right)^{\perp}$ be the multiset $\left\{\left(D^{0}\right)^{\perp} \mid D \in \Gamma\right\}$. The focused proofs of $\vdash\left(\Gamma^{0}\right)^{\perp}: \Uparrow R^{1}$ are in bijective correspondence with the LJ proofs of $\Gamma \vdash_{I} R$. 
For a detailed proof, see [16]. We simply illustrate one case in constructing the mapping from a collection of LLF rules to an LJ inference rule.

$$
\frac{\frac{\vdash\left(\Gamma^{0}\right)^{\perp},\left(D_{i}^{0}\right)^{\perp}: R^{1} \Uparrow}{\vdash\left(\Gamma^{0}\right)^{\perp}: R^{1} \Uparrow ?\left(D_{i}^{0}\right)^{\perp}}[?]}{\frac{\vdash\left(\Gamma^{0}\right)^{\perp}: R^{1} \Downarrow ?\left(D_{i}^{0}\right)^{\perp}}{\vdash\left(\Gamma^{0}\right)^{\perp}: R^{1} \Downarrow ?\left(D_{1}^{0}\right)^{\perp} \oplus ?\left(D_{2}^{0}\right)^{\perp}}[\oplus]} \quad \longmapsto \quad \frac{\Gamma, D_{i} \vdash_{I} R}{\Gamma, D_{1} \wedge D_{2} \vdash_{I} R}[\wedge L]
$$

The liberal use of ! in this translation throttles focusing. This translation is reminiscent of the earliest embedding of classical into intuitionistic logic of Kolmogorov, which uses the double negation in a similarly liberal fashion.

The $0 / 1$ translation can be used as a starting point in establishing the completeness of other proof systems. These systems can be seen as induced from alternative translations of intuitionistic logic.

Consider, for example, the $L J Q^{\prime}$ proof system presented in [7]. The translation for this system is given here using the " $q / j "$ mapping. The " $\otimes 1$ " device is another way to control focusing, or the lack thereof. All atoms must be given positive bias for this translation.

With minor changes, Girard's original (non-polarized) translation of intuitionistic logic [8] induces the complement to

\begin{tabular}{|c||c|c|}
\hline$F$ & $F^{q}$ (right) & $F^{j}$ (left) \\
\hline \hline atom $C$ & $C$ & $C$ \\
\hline false & 0 & 0 \\
\hline$A \wedge B$ & $A^{q} \otimes B^{q}$ & $! A^{j} \otimes ! B^{j}$ \\
\hline$A \vee B$ & $A^{q} \oplus B^{q}$ & $! A^{j} \oplus ! B^{j}$ \\
\hline$A \supset B$ & $\left(! A^{j} \multimap B^{q}\right) \otimes 1$ & $A^{q} \multimap ! B^{j}$ \\
\hline
\end{tabular}
$L J Q^{\prime}$ called $L J T$ [11], which is itself derived from LKT [5] (where this connection was noted implicitly.) All atoms must be given negative bias for this translation.

Given a translation such as that of $L J Q^{\prime}$, one can give a completeness proof for the system using a "grand tour" through linear logic as follows:

1. Show that a proof under the $0 / 1$ translation can be converted into a proof under the new translation. This usually follows from cut-elimination.

2. Define a mapping between proofs in the new system (such as LJQ) and LLF proofs of its translation.

3. Show soundness of the new system with respect to LJ. This is usually trivial. The "tour" is now complete, since proofs in LJ map to proofs under the 0/1 translation.

An intuitionistic system that contains atoms of both positive and negative bias is $\lambda$ RCC [13]. Two special cases of the $\supset L$ rule are distinguished involving $E \supset D$ for positive atom $E$ and $G \supset A$ for negative atom $A$. Each rule requires that the complementary atom ( $E$ on the left, $A$ on the right) is present when applied, thus terminating one branch of the proof. One can translate these special cases using forms $E \multimap ! D^{\prime}$ and $! G^{\prime} \multimap A$, respectively, in linear logic. The strategy outlined above can then be used to not only prove its completeness but also extend it with more aggressive focusing features. 
Our interest here is not the construction of individual systems but the building of a unifying framework for focusing in intuitionistic logic. Such a task requires a closer examination of polarity and its connection to focusing.

\section{Permeable Formulas and their Polarity}

Focused proofs in linear logic are characterized by two different phases: the invertible (asynchronous) phase and the non-invertible (synchronous) phase. These two phases are characterized by introduction rules for dual sets of formulas. In order to construct a general focusing scheme for intuitionistic logic, the nonlinear (exponential) aspects of proofs need special attention, especially in light of the fact that the [!] rule stops a bottom-up construction of focused application of synchronous rules (the arrow $\Downarrow$ in the conclusion flips to $\Uparrow$ in the premise).

For our purposes here, a particularly flexible way to deal with the exponentials in the translations of intuitionistic formulas is via the notion of permeation that is used in LU [10]. In particular, there are essentially three grades of permeation. The formula $B$ is left-permeable if $B \equiv ! B$, is right-permeable if $B \equiv ? B$, and neutral otherwise. Within sequent calculus proofs, a formula is left-permeable if it admits structural rules on the left and right-permeable if it admits structural rules on the right. An example of a left-permeable formula is $\exists x ! A$. All left-permeable formulas are synchronous and all right-permeables asynchronous. In the LU system, both the left and right sides of sequents contain two zones - one that treats formulas linearly and one that permits structural rules. A left-permeable (resp., right-permeable) formula is allowed to move between both zones on the left (right). In addition, LU introduces atoms that are inherently left or right-permeable or neutral. Although they appear to properly extend linear logic, one can simulate LU in "regular" linear logic by translating left-permeable atoms $A$ as ! $A$ and right-permeable ones as ? $A$.

To preserve the focusing characteristics of permeable atoms as positively or negatively biased atoms, we use the following LU-inspired asymmetrical translation. The superscript -1 indicates the left-side translation and +1 indicates the right-side translation:

$$
\begin{aligned}
& P^{-1}=! P \text { and } P^{+1}=P, \text { for left-permeable (positive) atom } P . \\
& N^{-1}=N \text { and } N^{+1}=? N, \text { for right-permeable (negative) atom } N . \\
& B^{-1}=B^{+1}=B, \text { for neutral atom } B .
\end{aligned}
$$

The ! rule of LLF causes a loss of focus in all circumstances, and is the main reason why we use an asymmetrical translation. The translation of positive atoms above preserves permeation on the left while allowing for focus on the right. That is, left-permeable atoms can now be interpreted meaningfully as positively biased atoms in focused proofs, and dually for right-permeable atoms. Furthermore, the permeation of positive atoms is "one-way only:" they cannot be selected for focus again once they enter the non-linear context.

Intuitionistic logic uses the left-permeable and neutral formulas and atoms. LU defines a translation for intuitionistic logic so that all synchronous formulas 
are left-permeable. For example, $\vee$ is translated as follows (here, $P, Q$ are positive and $N, M$ are negative $):(P \vee Q)^{-1}=P^{-1} \oplus Q^{-1},(P \vee N)^{-1}=P^{-1} \oplus ! N^{-1}$, $(N \vee P)^{-1}=! N^{-1} \oplus Q^{-1}$, and $(N \vee M)^{-1}=! N^{-1} \oplus ! M^{-1}$. The final element of intuitionistic polarity is that neutral atoms should be assigned negative bias in focused proofs. Neutral atoms that are introduced into the left context (e.g. by a $\supset L$ rule) must immediately end that branch of the proof in an identity rule. Otherwise, the unique stoup is lost when multiple non-permeable atoms accumulate in the linear context.

The LU and LLF systems serve as a convenient platform for the unified characterization of polarity and focusing in all three logics. We can now understand the terminology of "positive" and "negative" formulas in each logic as follows:

Linear logic: Positive formulas are synchronous formulas and positively biased neutral atoms. Negative formulas are asynchronous formulas and negatively biased neutral atoms.

Intuitionistic logic: Positive formulas are left-permeable formulas and negative formulas are asynchronous neutral formulas and negatively biased neutral atoms.

Classical logic: Positive formulas are left-permeable formulas. Negative formulas are right-permeable formulas.

\section{The LJF Sequent Calculus}

Since the polarities of intuitionistic logic observe stronger invariances, intuitionistic focused proofs are more well-structured than LLF proofs. The non-linear context of LLF contains both synchronous and asynchronous formulas, whereas in intuitionistic logic sequents can be clearly divided into zones respecting polarity. That is, when translating an intuitionistic sequent into a LLF sequent, synchronous formulas on the left are placed in the linear context.

We also make an adjustment on the LU translation of intuitionistic logic. Instead of using \& or $\otimes$ depending on the polarities of the subformulas, we construct two versions of intuitionistic conjunction, which has the following meaning in linear logic ( $P, Q$ for positives, $N, M$ for negatives, $A, B$ arbitrary):

$$
\begin{array}{lll}
\left(P \wedge^{+} Q\right)^{-1}=P^{-1} \otimes Q^{-1} & & \left(A \wedge^{+} B\right)^{+1}=A^{+1} \otimes B^{+1} \\
\left(P \wedge^{+} N\right)^{-1}=P^{-1} \otimes ! N^{-1} & & \\
\left(N \wedge^{+} P\right)^{-1}=! N^{-1} \otimes P^{-1} & & \left(A \wedge^{-} B\right)^{-1}=A^{-1} \& B^{-1} \\
\left(N \wedge^{+} M\right)^{-1}=! N^{-1} \otimes ! M^{-1} & & \left(A \wedge^{-} B\right)^{+1}=A^{+1} \& B^{+1}
\end{array}
$$

The connectives $\wedge^{-}$and $\wedge^{+}$are equivalent in intuitionistic logic in terms of provability but differ in their impact on the structure of focused proofs. The use of two conjunctions means that the top-level structure of formulas completely determines their polarity. Polarity in intuitionistic logic is defined as follows.

Definition 1. Atoms in LJF are arbitrarily positive or negative. Positive formulas are among positive atoms, true, false, $A \wedge^{+} B, A \vee B$ and $\exists x A$. Negative formulas are among negative atoms, $A \wedge^{-} B, A \supset B$ and $\forall x A$. 


$$
\begin{aligned}
& \frac{[N, \Gamma] \stackrel{N}{\longrightarrow}[R]}{[N, \Gamma] \longrightarrow[R]} \text { Lf } \quad \frac{[\Gamma]-{ }_{P} \rightarrow}{[\Gamma] \longrightarrow[P]} \text { Rf } \quad \frac{[\Gamma], P \longrightarrow[R]}{[\Gamma] \stackrel{P}{\longrightarrow}[R]} R_{l} \quad \frac{[\Gamma] \longrightarrow N}{[\Gamma]-N \rightarrow} R_{r} \\
& \frac{[C, \Gamma], \Theta \longrightarrow \mathcal{R}}{[\Gamma], \Theta, C \longrightarrow \mathcal{R}}[]_{l} \quad \frac{[\Gamma], \Theta \longrightarrow[D]}{[\Gamma], \Theta \longrightarrow D}[]_{r} \\
& \overline{[P, \Gamma]-P \rightarrow} I_{r}, \text { atomic } P \quad \overline{[\Gamma] \stackrel{N}{\longrightarrow}[N]} I_{l} \text {, atomic } N \\
& \frac{[\Gamma], \Theta \longrightarrow \mathcal{R}}{[\Gamma], \Theta, \text { false } \longrightarrow \mathcal{R}} \text { falseL } \quad \frac{[\Gamma], \Theta, \text { true } \longrightarrow \mathcal{R}}{\text { true }} \quad \overline{[\Gamma]-\text { true }^{\prime}} \text { true } R \\
& \frac{[\Gamma] \stackrel{A_{i}}{\longrightarrow}[R]}{[\Gamma] \stackrel{A_{1} \wedge^{-} A_{2}}{\longrightarrow}[R]} \wedge^{-} L \quad \frac{[\Gamma], \Theta, A, B \longrightarrow \mathcal{R}}{[\Gamma], \Theta, A \wedge^{+} B \longrightarrow \mathcal{R}} \wedge^{+} L \\
& \frac{[\Gamma], \Theta \longrightarrow A \quad[\Gamma], \Theta \longrightarrow B}{[\Gamma], \Theta \longrightarrow A \wedge^{-} B} \wedge^{-} R \quad \frac{[\Gamma]-{ }_{A} \rightarrow[\Gamma]-{ }_{B} \rightarrow}{[\Gamma]-{ }_{A} \wedge^{+} B} \wedge^{+} R \\
& \frac{[\Gamma], \Theta, A \longrightarrow \mathcal{R} \quad[\Gamma], \Theta, B \longrightarrow \mathcal{R}}{[\Gamma], \Theta, A \vee B \longrightarrow \mathcal{R}} \vee L \quad \frac{[\Gamma]-A_{i} \rightarrow}{[\Gamma]-A_{1} \vee A_{2} \rightarrow} \vee R \\
& \frac{[\Gamma]-{ }_{A} \rightarrow[\Gamma] \stackrel{B}{\longrightarrow}[R]}{[\Gamma] \stackrel{A \supset B}{\longrightarrow}[R]} \supset L \quad \frac{[\Gamma], \Theta, A \longrightarrow B}{[\Gamma], \Theta \longrightarrow A \supset B} \supset R \\
& \frac{[\Gamma], \Theta, A \longrightarrow \mathcal{R}}{[\Gamma], \Theta, \exists y A \longrightarrow \mathcal{R}} \exists L \quad \frac{[\Gamma]-{ }_{A[t / x]} \rightarrow}{[\Gamma]-\exists_{x} \rightarrow} \exists R \quad \frac{[\Gamma] \stackrel{A[t / x]}{\longrightarrow}[R]}{[\Gamma] \stackrel{\forall x A}{\longrightarrow}[R]} \forall L \quad \frac{[\Gamma], \Theta \longrightarrow A}{[\Gamma], \Theta \longrightarrow \forall y A} \forall R
\end{aligned}
$$

Fig. 2. The Intuitionistic Sequent Calculus LJF. Here, $P$ is positive, $N$ is negative, $C$ is a negative formula or positive atom, and $D$ a positive formula or negative atom. Other formulas are arbitrary. Also, $y$ is not free in $\Gamma, \Theta$, or R.

The above translation induces the sequent calculus $L J F$ for intuitionistic logic, shown in Figure 2. Sequents in $L J F$ can be interpreted as follows:

1. $[\Gamma], \Theta \longrightarrow \mathcal{R}$ (end sequent): this is an unfocused sequent. $\Gamma$ contains negative formulas and positive atoms. $\mathcal{R}$ represents either a formula $R$ or $[R]$.

2. $[\Gamma] \longrightarrow[R]$ : this represents a sequent in which all asynchronous formulas have been decomposed, and is ready for a formula to be selected for focus.

3. $[\Gamma] \stackrel{A}{\longrightarrow}[R]:$ this is a left-focusing sequent, with focus on formula $A$. The meaning of this sequent remains $\Gamma, A \vdash_{I} R$.

4. $[\Gamma]-A_{A} \rightarrow$ : this is a right-focusing sequent on formula $A$, with the meaning $\Gamma \vdash_{I} A$.

Theorem 1. LJF is sound and complete with respect to intuitionistic logic.

Proof. Using the "grand tour" strategy. See [16, Section 6] for details. 
Given the different forms of sequents, the cut rule for $L J F$ takes many forms:

$$
\begin{gathered}
\frac{[\Gamma], \Theta \longrightarrow P \quad\left[\Gamma^{\prime}\right], \Theta^{\prime}, P \longrightarrow \mathcal{R}}{\left[\Gamma \Gamma^{\prime}\right], \Theta \Theta^{\prime} \longrightarrow \mathcal{R}} C u t^{+} \quad \frac{[\Gamma], \Theta \longrightarrow C \quad\left[C, \Gamma^{\prime}\right], \Theta^{\prime} \longrightarrow \mathcal{R}}{\left[\Gamma \Gamma^{\prime}\right], \Theta \Theta^{\prime} \longrightarrow \mathcal{R}} C^{\longrightarrow} \\
\stackrel{[\Gamma] \stackrel{B}{\longrightarrow}[P]\left[\Gamma^{\prime}\right], P \longrightarrow[R]}{\left[\Gamma \Gamma^{\prime}\right] \stackrel{B}{\longrightarrow}[R]} C u t_{1}^{\leftarrow} \frac{[\Gamma] \longrightarrow N \quad\left[N, \Gamma^{\prime}\right] \stackrel{B}{\longrightarrow}[R]}{\left[\Gamma \Gamma^{\prime}\right] \stackrel{B}{\longrightarrow}[R]} C u t_{2}^{\leftarrow} \\
\frac{[\Gamma]-{ }_{C} \rightarrow\left[C, \Gamma^{\prime}\right]-{ }_{R} \rightarrow}{\left[\Gamma \Gamma^{\prime}\right]-{ }_{R} \rightarrow} C u t^{\rightarrow}
\end{gathered}
$$

Notice that the last three cut rules retain focus in the conclusion. These rules extend those of $L J Q^{\prime}$ [7], which were shown to be useful for studying termreduction systems. See [16] for a proof of the admissibility of these rules.

Like LLF, a key characteristic of $L J F$ is the assignment of arbitrary polarity to atoms. To illustrate the effect of these assignments on the structure of focused proofs, consider the sequent $a, a \supset b, b \supset c \vdash c$ where $a, b$ and $c$ are atoms. This sequent can be proved either by forward chaining through the clause $a \supset b$, or backward chaining through the clause $b \supset c$. Assume that atoms $a$ and $b$ are assigned positive polarity and that $c$ is assigned negative polarity. This assignment effectively adopts the forward chaining strategy, reflected in the following $L J F$ proof segment (here, $\Gamma$ is the set $\{a, a \supset b, b \supset c\}$ ):

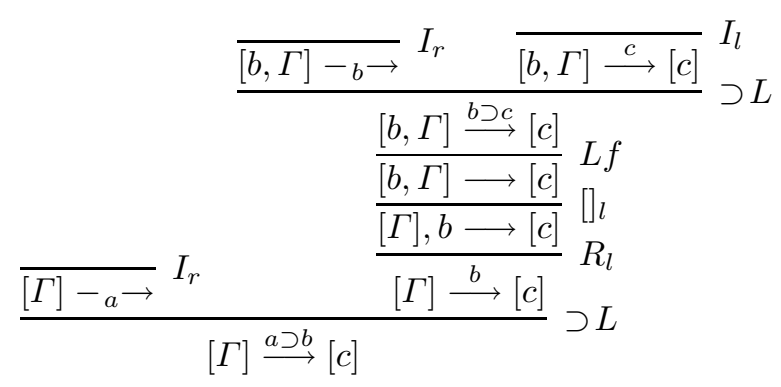

The polarities of $a$ and $c$ do not fundamentally affect the structure of the proof in this example. However, assigning negative polarity to atom $b$ would restrict the proof to use the backward chaining strategy:

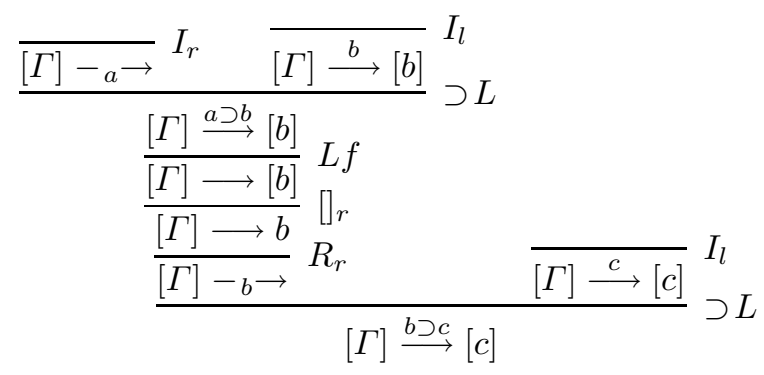




\section{Embedding Intuitionistic Systems in LJF}

The LJF proof system can be used to "host" other focusing proof system for intuitionistic logic. One obvious restriction to LJF is its purely negative fragment, which essentially corresponds to LJT. In the negative fragment one also finds uniform proofs, where the right "goal" formula is always fully decomposed before any left rule is applied. Various other proof systems can be embedded into $L J F$ by mapping intuitionistic formulas to intuitionistic formulas in such a way that focusing features in $L J F$ are stopped by the insertion of delay operators. In particular, if we define $\partial^{-}(B)=$ true $\supset B$ and $\partial^{+}(B)=$ true $\wedge^{+} B$, then $B$, $\partial^{-}(B)$, and $\partial^{+}(B)$ are all logically equivalent but $\partial^{-}(B)$ is always negative and $\partial^{+}(B)$ is always positive.

Proofs in the LJQ' system can be embedded into LJF by translating all left-side formulas $(l)$ as negatives and all right-side formulas $(r)$ as positives: in particular, for atom $\mathrm{B}, B^{l}=B^{r}=B$, false ${ }^{l}=\partial^{-}$(false), false $e^{r}=$ false, $(A \wedge B)^{l}=\partial^{-}\left(A^{l} \wedge^{+} B^{l}\right),(A \wedge B)^{r}=A^{r} \wedge^{+} B^{r},(A \vee B)^{l}=\partial^{-}\left(A^{l} \vee B^{l}\right)$, $(A \vee B)^{r}=A^{r} \vee B^{r},(A \supset B)^{l}=A^{r} \supset \partial^{+}\left(B^{l}\right),(A \supset B)^{r}=\partial^{+}\left(A^{l} \supset B^{r}\right)$.

Arbitrary LJ proofs can be embedded within LJF by inserting sufficient delaying operators. The table here provides the translation (redefining the superscripts $l$ and $r$, for convenience). Together with cutelimination, the embedding also suggests a completeness proof for LJF independently of linear logic. The following example embeds the $\wedge R$ rule in $L J F$ :

\begin{tabular}{|c||c|c|}
\hline$F$ & $F^{l}$ (left) & $F^{r}$ (right) \\
\hline \hline atom $C$ & $C$ & $C$ \\
\hline false & $\partial^{-}$(false) & false \\
\hline true & $\partial^{-}($true $)$ & true \\
\hline$A \wedge B$ & $\partial^{+}\left(A^{l}\right) \wedge^{-} \partial^{+}\left(B^{l}\right)$ & $\partial^{+}\left(A^{r} \wedge^{-} B^{r}\right)$ \\
\hline$A \vee B$ & $\partial^{-}\left(A^{l} \vee B^{l}\right)$ & $\partial^{-}\left(A^{r}\right) \vee \partial^{-}\left(B^{r}\right)$ \\
\hline$A \supset B$ & $\partial^{-}\left(A^{r}\right) \supset \partial^{+}\left(B^{l}\right)$ & $\partial^{+}\left(A^{l} \supset B^{r}\right)$ \\
\hline$\exists x A$ & $\partial^{-}\left(\exists x A^{l}\right)$ & $\exists x \partial^{-}\left(A^{r}\right)$ \\
\hline$\forall x A$ & $\forall x \partial^{+}\left(A^{l}\right)$ & $\partial^{+}\left(\forall x A^{r}\right)$ \\
\hline
\end{tabular}

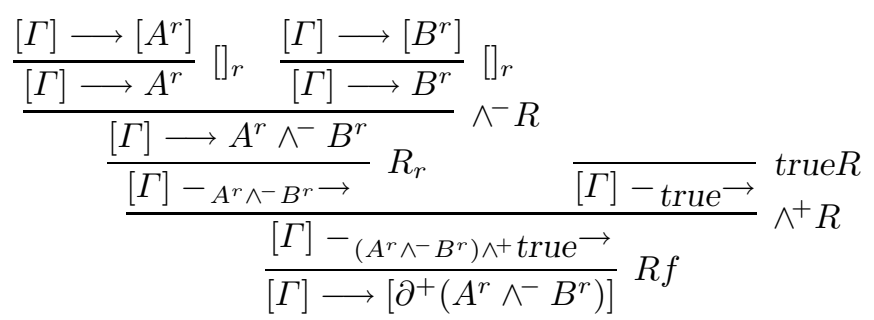

The system $\lambda \mathrm{RCC}$ also presents interesting choices. In particular, it may not always be the best choice to focus maximally. Forward chaining may generate a new formula or "clause" that may need to be used multiple times. In a $\supset L$ rule on formulas $E \supset D$ where $E$ is a positive atom, one may not wish to decompose the formula $D$ immediately. This is accomplished in the linear translation with a !. It can also be accomplished by using formulas $E \supset \partial^{+}(D)$ in case $D$ is negative, and $E \supset \partial^{+}\left(\partial^{-}(D)\right)$ in case $D$ is positive. Note that unlike the $l / r$ translations for LJQ and LJ above, these simple devices do not hereditarily alter the structure of $D$. 


\section{Embedding Classical Logic in LJF}

We can use $L J F$ to formulate a focused sequent calculus for classical logic that reveals the latter's constructive content in the style of $\mathrm{LC}$. While it is possible to derive such a system again using linear logic, classical logic can also be embedded within intuitionistic logic using the double-negation translations of Gödel, Gentzen, and Kolmogorov. These translations do not, however, yield significant focusing features. Girard's polarized version of the double negation translation for LC approaches the problem of capturing duality in a more subtle way. Following the style of $L J F$, we wish to define dual versions of each propositional connective, which leads to a more usable calculus. We thus modify the LC translation in a natural way, which is consistent with its original intent. The proof system we derive is called $L K F$.

We must first separate classical from intuitionistic polarity since these are different notions (see the end of Section 4).

Definition 2. Atoms are arbitrarily classified as either positive or negative. The literal $\neg A$ has the opposite polarity of the atom $A$. Positive formulas are among positive literals, $\mathcal{T}, \mathcal{F}, A \wedge^{+} B, A \vee^{+} B, A \supset^{+} B$ and $\exists x A$. Negative formulas are among negative literals, $\neg \mathcal{T}, \neg \mathcal{F}, A \wedge^{-} B, A \vee^{-} B, A \supset^{-} B$ and $\forall x A$. Negation $\neg A$ is defined by de Morgan dualities $\neg A / A, \wedge^{+} / \vee^{-}, \wedge^{-} / \mathcal{}^{+}$and $\forall / \exists$. Negative implication $A \supset^{-} B$ is defined as $\neg A \vee^{-} B$ and $A \supset^{+} B$ is defined as $\neg A \vee^{+} B$. Formulas are assumed to be in negation normal form (that is, formulas that do not contain implications and negations have atomic scope).

The constants $\mathcal{T}, \mathcal{F}, \neg \mathcal{T}$ and $\neg \mathcal{F}$ are best described, respectively, as 1,0 , $\perp$ and $T$ in linear logic. Just as we have dual versions of each connective, we also have dual versions of each identity. But this is not linear logic as the formulas are polarized in the extreme. The distinction between the positive and negative versions of each connective affects only the structure of proofs and not provability.

Let $\sim A$ represent the intuitionistic formula $A \supset \phi$ where $\phi$ is some unspecified positive atom. The " $\approx$ " embedding of classical logic is found in Table 2. Variations are possible on the embedding. Note that the classical $\wedge^{-}$is not defined in terms of the intuitionistic $\wedge^{-}$. The embeddings are selected to enforce the dualities $\wedge^{-} \mathrm{N}^{+}$and $\wedge^{+} / \mathrm{V}^{-}$. Alternatives may also work, but will increase the complexity of the derivation. Here, the cases all follow the pattern $P$ or $\sim P$ where $P$ is a positive intuitionistic formula. In particular, negative intuitionistic atoms are not used in the embedding.

The $\approx$ embedding induces the $L K F$ sequent calculus in Figure 3 from the image of $L J F$ proofs, analogous to how $L J F$ was derived from LLF. Here is one sample correspondence between a $L J F$ rule and a LKF rule:

$$
\frac{[\Delta], \Psi, A, B \longrightarrow[\phi]}{[\Delta], \Psi, A \wedge^{+} B \longrightarrow[\phi]} \wedge^{+} L \quad \longmapsto \quad \frac{\vdash[\Theta], \Gamma, A, B}{\vdash[\Theta], \Gamma, A \vee^{-} B} \vee^{-}
$$

Sequents of the form $\vdash[\Theta], \Gamma$ are unfocused while those of the form $\mapsto[\Theta], A$ focus on the stoup formula $A$. 


\begin{tabular}{|c|c|c|c|c|c|c|c|c|c|c|}
\hline \multicolumn{2}{|c|}{\begin{tabular}{|l|l|}
$\mathcal{A}^{\approx}$ & $\mathcal{B}^{\approx}$ \\
\end{tabular}} & \multicolumn{2}{|c|}{$\left(\mathcal{A} \wedge^{+} \mathcal{B}\right) \approx$} & \multicolumn{2}{|c|}{$\left(\mathcal{A} \wedge^{-} \mathcal{B}\right)^{\approx}$} & \multicolumn{2}{|c|}{$\left(\mathcal{A} \vee^{+} \mathcal{B}\right)^{\approx}$} & \multicolumn{2}{|c|}{$(\mathcal{A} \vee \mathcal{B}) \approx$} & $(\neg \mathcal{A})^{2}$ \\
\hline 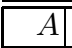 & $B$ & \multicolumn{2}{|c|}{$\overline{A A \wedge^{+} B}$} & \multicolumn{2}{|c|}{$\sim \sim(\sim A \vee \sim B)$} & \multicolumn{2}{|c|}{$A \vee B$} & \multicolumn{2}{|c|}{$\sim \sim\left(\sim A \wedge^{+} \sim B\right) \mid$} & $\bar{\sim} \sim A$ \\
\hline$A$ & $\sim B$ & \multicolumn{2}{|c|}{$A \wedge^{+} \sim B$} & \multicolumn{2}{|c|}{$\sim(\sim A \vee B)$} & \multicolumn{2}{|c|}{$A \vee \sim B$} & \multicolumn{2}{|c|}{$\sim\left(\sim A \wedge^{+} B\right)$} & \\
\hline$\sim A$ & $B$ & \multicolumn{2}{|c|}{$\sim A \wedge^{+} B$} & \multicolumn{2}{|c|}{$\sim(A \vee \sim B)$} & \multicolumn{2}{|c|}{$\sim A \vee B$} & \multicolumn{2}{|c|}{$\sim\left(A \wedge^{+} \sim B\right)$} & $A$ \\
\hline \begin{tabular}{|l|}
$\sim A$ \\
\end{tabular} & $\checkmark B$ & \multicolumn{2}{|c|}{$\sim A \wedge^{+} \sim B$} & \multicolumn{2}{|c|}{$\sim(A \vee B)$} & \multicolumn{2}{|c|}{$\sim A \vee \sim B$} & \multicolumn{2}{|c|}{$\sim\left(A \wedge^{+} B\right)$} & \\
\hline & $\mathcal{A}^{\approx}$ & $\mathcal{B}^{\approx}$ & $\left(\mathcal{A} \supset^{\prime}\right.$ & $\mathcal{B}) \approx$ & $(\mathcal{A})^{-}$ & $\mathcal{B}) \approx$ & & $x \mathcal{A})^{\approx}$ & $(\exists x \mathcal{A}) \approx$ & \\
\hline & $A$ & $B$ & $\sim A$ & $B$ & $\sim\left(A \wedge^{+}\right.$ & $+\sim B)$ & & $\exists x \sim A)$ & $\exists x A$ & \\
\hline & $A$ & $A \sim B$ & $\sim A \vee$ & $\sim B$ & $\sim(A /$ & $\overline{\left.\Lambda^{+} B\right)}$ & & . & . & \\
\hline & $\sim A$ & $B$ & $A \vee$ & $B$ & $\sim(\sim$ & $\sim B)$ & & $(\exists x A)$ & $\exists x \sim A$ & \\
\hline & $\sim A$ & $4 \sim B$ & $A \vee$ & $\sim B$ & $\sim(\sim A$ & $\wedge^{+} B$ & & . & . & \\
\hline
\end{tabular}

Table 2. Polarized embedding of classical logic. The $(\cdot) \approx$ translation on compound formulas is given above (there, $A, B$ represent formulas not preceded by $\sim$ ). For positive classical atom $P, P^{\approx}=P$; for negative classical atom $N, N^{\approx}=\sim N$; (where both $P$ and $N$ are assigned positive intuitionistic polarity), and for the logical constants $\mathcal{T}^{\approx}=$ true, $\mathcal{F} \approx=$ false,$(\neg \mathcal{T}) \approx=\sim$ true,$(\neg \mathcal{F}) \approx=\sim$ false.

The following correctness theorem for LKF can be proved by relating it to the Gödel-Gentzen translation (see [16, Section 9] for more details).

Theorem 2. LKF is sound and complete with respect to classical logic.

We have constructed this embedding of classical logic as a further demonstration of the abilities of $L J F$ as a hosting framework. The embedding also revealed interesting relationships between classical and intuitionistic polarity. It is also possible to derive LKF from linear logic: each connective needs to be defined as either wholly positive or negative. For example, the translation of $\left(A \vee^{-} B\right)^{p}$ is $A^{p} 28 B^{p}$ if $A^{p}$ and $B^{p}$ are both negative; is $A^{p} \approx 8$ ? $B^{p}$ if only $A^{p}$ is negative; is ? $A^{p}>8 B^{p}$ if only $B^{p}$ is negative; and is ? $A^{p} \not 8 ? B^{p}$, if $A^{p}$ and $B^{p}$ are both positive. This translation is called the "polaro" translation in [6], where it was used to formulate $L K_{p}^{\eta}$, the first focused proof system for classical logic. Like the $\approx$ translation, the polaro translation is a derivative of the $\mathrm{LC} / \mathrm{LU}$ analysis of polarity. Except for the treatment of atoms, LKF is derivable from LLF using the polaro translation in the same manner that LJF is derived.

$L K_{p}^{\eta}$ was extended to $L K_{p o l}^{\eta, \rho}$ in [14]. These systems were formulated independently of Andreoli's results. The authors of [6] opted not to present $L K_{p}^{\eta}$ as a sequent calculus because they feared that it will have the cumbersome size of LU. Such cumbersomeness can, in fact, be avoided by adopting LLF-style reaction rules.

Given our goals, the choice in adopting Andreoli's system is justified in that LKF and LJF have the form of compact sequent calculi ready for implementation. More significantly perhaps, $L K_{p}^{\eta}$ and $L K_{\text {pol }}^{\eta, \rho}$ define focusing for classical logic. They map to polarized forms of linear logic (LLP and LL $\left.\mathrm{Lol}_{\text {pl }}\right)$. LLF is defined for full classical linear logic. LKF is embedded within LLF in the same way that LC is embedded within LU. LLF is well suited for hosting other logics. 


$$
\begin{aligned}
& \frac{\vdash[\Theta, C], \Gamma}{\vdash[\Theta], \Gamma, C}[] \quad \frac{\mapsto[P, \Theta], P}{\vdash[P, \Theta]} \text { Focus } \quad \frac{\vdash[\Theta], N}{\mapsto[\Theta], N} \text { Release } \\
& \overline{\mapsto[\neg P, \Theta], P} I D^{+} \text {, atomic } P \quad \overline{\mapsto[N, \Theta], \neg N} I D^{-} \text {, atomic } N \\
& \overline{\mapsto[\Theta], \mathcal{T}} \text { indeed } \quad \overline{\vdash[\Theta], \Gamma, \neg \mathcal{F}} \text { absurd } \frac{\vdash[\Theta], \Gamma}{\vdash[\Theta], \Gamma, \neg \mathcal{T}} \text { trivial } \\
& \frac{\vdash[\Theta], \Gamma, A \vdash[\Theta], \Gamma, B}{\vdash[\Theta], \Gamma, A \wedge^{-} B} \wedge^{-} \quad \frac{\vdash[\Theta], \Gamma, A, B}{\vdash[\Theta], \Gamma, A \vee B} \vee \\
& \frac{\vdash[\Theta], \Gamma, B, \neg A}{\vdash[\Theta], \Gamma, A \supset^{-} B} \supset^{-} \quad \frac{\vdash[\Theta], \Gamma, A}{\vdash[\Theta], \Gamma, \forall x A} \forall \\
& \frac{\mapsto[\Theta], A \mapsto[\Theta], B}{\mapsto[\Theta], A \wedge^{+} B} \wedge^{+} \quad \frac{\mapsto[\Theta], A_{i}}{\mapsto[\Theta], A_{1} \vee^{+} A_{2}} \vee^{+} \quad \frac{\mapsto[\Theta], A[t / x]}{\mapsto[\Theta], \exists x A} \exists \\
& \frac{\mapsto[\Theta], \neg A}{\mapsto[\Theta], A \supset^{+} B} \supset^{+} \quad \frac{\mapsto[\Theta], B}{\mapsto[\Theta], A \supset^{+} B} \supset^{+}
\end{aligned}
$$

Fig. 3. The Classical Sequent Calculus LKF. Here, $P$ is positive, $N$ is negative, $C$ is a positive formula or a negative literal, $\Theta$ consists of positive formulas and negative literals, and $x$ is not free in $\Theta, \Gamma$. End-sequents have the form $\vdash$ [], $\Gamma$.

\section{Conclusion and Future Work}

We have studied focused proof construction in intuitionistic logic. The key to this endeavor is the definition of polarity for intuitionistic logic. The LJF proof system captures focusing using this notion of polarity. We illustrate how systems such as LJ, LJT, LJQ, and $\lambda$ RCC can be captured within LJF by assigning polarity to atoms and by adding to intuitionistic logic formulas annotations on conjunctions and delaying operators. We also use LJF to derive and justify the $L K F$ focusing proof system for classical logic.

It remains to examine the impact of these focusing calculi on typed $\lambda$-calculi, logic programming, and theorem proving. Given the connections observed between LJQ/LJT and call-by-name/value, the LJF system could provide a framework for $\lambda$-term evaluations that combine the eager and lazy evaluation strategies. In the area of theorem proving, there are a number of completeness theorems for various restrictions to resolution: it would be interesting to see if any of these are captured by an appropriate mapping into $L K F$.

Acknowledgments We would like to thank the reviewers of an earlier version of this paper for their comments. The work reported here was carried out while the first author was on sabbatical leave from Hofstra University to LIX. The second author was supported by INRIA through the "Equipes Associées" Slimmer and by the Information Society Technologies program of the European Commission, Future and Emerging Technologies under the IST-2005-015905 MOBIUS project. 


\section{References}

1. Jean-Marc Andreoli. Logic programming with focusing proofs in linear logic. J. of Logic and Computation, 2(3):297-347, 1992.

2. Kaustuv Chaudhuri. The Focused Inverse Method for Linear Logic. PhD thesis, Carnegie Mellon University, December 2006. Technical report CMU-CS-06-162.

3. Kaustuv Chaudhuri, Frank Pfenning, and Greg Price. A logical characterization of forward and backward chaining in the inverse method. In U. Furbach and N. Shankar, editors, Proceedings of the 3rd International Joint Conference on Automated Reasoning (IJCAR'06), LNCS 4130, pp. 97-111. Springer, August 2006.

4. Pierre-Louis Curien and Hugo Herbelin. The duality of computation. In ICFP '00: Proceedings of the fifth ACM SIGPLAN international conference on Functional programming, pages 233-243, New York, NY, USA, 2000. ACM Press.

5. Vincent Danos, Jean-Baptiste Joinet, and Harold Schellinx. LKQ and LKT: sequent calculi for second order logic based upon dual linear decompositions of classical implication. In Girard, Lafont, and Regnier, editors, Workshop on Linear Logic, pages 211-224. London Mathematical Society Lecture Notes 222, Cambridge University Press, 1995.

6. Vincent Danos, Jean-Baptiste Joinet, and Harold Schellinx. A new deconstructive logic: Linear logic. Journal of Symbolic Logic, 62(3):755-807, 1997.

7. R. Dyckhoff and S. Lengrand. LJQ: a strongly focused calculus for intuitionistic logic. In A. Beckmann et al, editor, Computability in Europe 2006, LNCS 3988, pages 173-185. Springer Verlag, 2006.

8. Jean-Yves Girard. Linear logic. Theoretical Computer Science, 50:1-102, 1987.

9. Jean-Yves Girard. A new constructive logic: classical logic. Math. Structures in Comp. Science, 1:255-296, 1991.

10. Jean-Yves Girard. On the unity of logic. Annals of Pure and Applied Logic, 59:201-217, 1993.

11. Hugo Herbelin. Séquents qu'on calcule: de l'interprétation du calcul des séquents comme calcul de lambda-termes et comme calcul de stratégies gagnantes. PhD thesis, Université Paris 7, 1995.

12. J. M. Howe. Proof Search Issues in Some Non-Classical Logics. PhD thesis, University of St Andrews, December 1998. Available as University of St Andrews Research Report CS/99/1.

13. Radha Jagadeesan, Gopalan Nadathur, and Vijay Saraswat. Testing concurrent systems: An interpretation of intuitionistic logic. In Proceedings of FSTTCS, 2005.

14. Olivier Laurent, Myriam Quatrini, and Lorenzo Tortora de Falco. Polarized and focalized linear and classical proofs. Ann. Pure Appl. Logic, 134(2-3):217-264, 2005.

15. P. B. Levy. Jumbo lambda-calculus. In M. Bugliesi, B. Preneel, V. Sassone, and I. Wegener, editors, ICALP06: 33rd International Colloquium on Automata, Languages and Programming, LNCS 4052. Springer, July 2006.

16. Chuck Liang and Dale Miller. On focusing and polarities in linear logic and intuitionistic logic. Unpublished report, December 2006.

17. Dale Miller, Gopalan Nadathur, Frank Pfenning, and Andre Scedrov. Uniform proofs as a foundation for logic programming. Annals of Pure and Applied Logic, 51:125-157, 1991.

18. Frank Pfenning. Automated theorem proving. Lecture notes, March 2004. 